\title{
On the Optimal Detection and Error Performance Analysis of the Hardware Impaired Systems
}

\author{
Sidrah Javed ${ }^{1}$, Osama Amin ${ }^{1}$, Salama S. Ikki ${ }^{2}$ and Mohamed-Slim Alouini ${ }^{1}$ \\ ${ }^{1}$ King Abdullah University of Science and Technology (KAUST), Thuwal, Makkah Province, Saudi Arabia. \\ E-mail: $\{$ sidrah.javed, osama.amin, slim.alouini\}@kaust.edu.sa \\ ${ }^{2}$ Department of Electrical Engineering, Faculty of Engineering, Lakehead University, Ontario, Canada. \\ E-mail: sikki@lakeheadu.ca
}

\begin{abstract}
The conventional minimum Euclidean distance (MED) receiver design is based on the assumption of ideal hardware transceivers and proper Gaussian noise in communication systems. Throughout this study, an accurate statistical model of various hardware impairments (HWIs) is presented. Then, an optimal maximum likelihood (ML) receiver is derived considering the distinct characteristics of the HWIs comprised of additive improper Gaussian noise and signal distortion. Next, the average error probability performance of the proposed optimal ML receiver is analyzed and tight bounds are derived. Finally, different numerical and simulation results are presented to support the superiority of the proposed ML receiver over MED receiver and the tightness of the derived bounds.
\end{abstract}

\section{INTRODUCTION}

The rapidly growing demand for Internet of Things (IoT) has imposed tremendous design challenges for the modern wireless communication systems such as compactness, least complexity, cost effectiveness and high energy efficiency. Immense research has been carried out to develop new techniques/configurations to tackle the design challenges e.g. direct conversion transceivers have recently become an active research area [1], [2]. However, the performance of the proposed systems can be highly affected by the non-ideal operation of radio frequency (RF) transceivers. Hardware impairments (HWIs) impose a huge challenge on next-generation network planning and deployment [3], [4]. Specifically, they can limit the deployment of high frequency circuitry [5], [6]. HWIs emerge in various RF stages including imperfections in analog-to-digital/digital-to-analog converters, non-linear high power amplifier/low noise amplifier, mismatched local oscillator and phase shifter, etc. [6], [7]. HWIs not only result in phase/amplitude errors and raised noise floor but also render inevitable mixing of the image and desired signals. The distinct behavior of the HWIs motivates us to develop accurate models and propose effective compensation techniques to meet the expected performance.

Some research has been carried out to precisely model these HWIs and analyze the impaired system performance. For example, [6] studies the impact and digital compensation of RF imperfections in high-rate wireless systems in great detail. A novel data-detection algorithm for transmitter impaired MIMO systems is proposed in [8]. The rate maximization under improper additive distortions has been investigated extensively in the literature [9], [10]. However, error probability performance under improper interference is the least explored dimension. Only a few work such as, [11] studies the error probability performance under improper interference with asymmetric transmission. However, to the best of author knowledge, this paper is the first to address the accurate statistical model of HWI system, optimal detection scheme and the practical problem of average error probability, considering both transmitter and receiver impairments with given signal modulations.

In this paper, we analyze the performance of a simple communication system suffering from HWIs represented mainly in in-phase and quadrature (I/Q) imbalance and additive distortions. Section II describes the adopted system along with its complete characterization. In section III, we propose an optimal maximum likelihood (ML) detector for the system under mentioned HWIs. Next, we present error probability analysis based on the pairwise error probability for generalization of any higher order M-ary modulation scheme in section IV. Later average probability of error bounds are computed for three different scenarios in section $\mathrm{V}$, followed by the numerical results to validate the derived bounds and analyze the performance gain reaped by adopting optimal ML detector in place of sub-optimal MED detector.

Notations: In this paper, $|a|$ and $a^{*}$ represent the absolute and complex conjugate of a scalar complex number $a$. The probability of an event $A$ is defined as $\operatorname{Pr}(A)$. The notations $f_{z}(z)$ and $f_{z \mid y}(z \mid y)$ denote the probability density function (PDF) of random variable $z$ and conditional PDF of $z$ given $y$. In addition, $\xi_{r}$ and $\xi_{i}$ depict the real and imaginary components of a complex variable $\xi$. Similarly, we use $\sigma_{I}^{2}$ and $\sigma_{Q}^{2}$ to illustrate the variance of the in-phase and quadrature component. We use $g^{(n)}(\bar{x})$ for the $n^{\text {th }}$ derivative of function $g(x)$ w.r.t $x$ evaluated at $\bar{x}$. Lastly, $n$ ! presents the factorial operator of a non-negative integer $n$.

\section{SYSTEM DESCRIPTION}

\section{A. Preliminaries}

To characterize the difference between the proper Gaussian signaling (PGS) and improper Gaussian signaling (IGS) schemes, we consider a zero-mean complex Gaussian random variable $x \in \mathbb{C}$ and introduce the following definitions:

Definition 1. The variance and the pseudo-variance of $x$ are defined, respectively, as $\sigma_{x}^{2}=\mathrm{E}\left[|x|^{2}\right]$ and $\tilde{\sigma}_{x}^{2}=\mathrm{E}\left[x^{2}\right]$, where $\mathrm{E}[$.$] is the expected value operator [12].$

Definition 2. A complex random variable is called proper if its pseudo-variance is zero, otherwise it is called improper [12].

Definition 3. The complex Gaussian random variable $x$ is described as, $x \sim \mathcal{C N}\left(m_{x}, \sigma_{x}^{2}, \tilde{\sigma}_{x}^{2}\right)$, where $m_{x}$ is its mean. 


\section{B. Hardware Impaired Signal Model}

Consider a wireless communication system employing synchrodyne architecture transceivers in the RF front-end [13]. At the transmitter, the digital baseband modulated signal undergoes pulse shaping and up-conversion to the desired carrier frequency. During this homodyne up-conversion stage, the imperfect local oscillator and phase shifter introduce I/Q imbalance. Transceivers with I/Q imbalance not only contribute to the amplitude and phase errors but also induce widely linear transformation (WLT) of the modulated signal $x_{m}$. This transformation results in a self-interfering (SI) signal $x_{\mathrm{IQI}}$, due to the limited image-rejection capability [6], expressed as,

$$
x_{\mathrm{IQI}}=\nu_{1} x_{m}+\nu_{2} x_{m}^{*} ; \quad m=1,2, \ldots, M,
$$

where $x_{m}$ is the bandpass modulated signal taken from MQAM, M-PSK or M-PAM constellation in the ideal scenario, which has been transformed to $x_{\mathrm{IQI}}$ pertaining to the transmitter I/Q imbalance. In addition, $\nu_{1}$ and $\nu_{2}$ account for the amplitude $\left(a_{\mathrm{t}}\right)$ and phase $\left(\theta_{\mathrm{t}}\right)$ errors at the transmitter I/Q mixer stage and are generally modeled as

$$
\nu_{1}=\left(1+a_{\mathrm{t}} e^{j \theta_{\mathrm{t}}}\right) / 2 ; \nu_{2}=1-\nu_{1}^{*}=\left(1-a_{\mathrm{t}} e^{-j \theta_{\mathrm{t}}}\right) / 2
$$

It is important to highlight that the ideal transmitter I/Q mixer stage is assumed to exhibit $a_{\mathrm{t}}=1$ and $\theta_{\mathrm{t}}=0$ amplitude and phase translation respectively. This implies that the bandpass modulated signal is given by $x_{\mathrm{IQI}}=x_{m}$, under ideal circumstances. Moreover, non-linear transfer function of various transmitter RF stages, such as digital-to-analog converter, band-pass filter and high power amplifier result in accumulative additive distortion noise raising the noise floor [6]. This additive distortion is modelled as a zero-mean proper complex Gaussian random variable $d_{\mathrm{t}} \sim \mathcal{C N}\left(0, \sigma_{\mathrm{t}}^{2}, 0\right)$ [14]. Thus the transmitted signal reduces to

$$
x_{\mathrm{tx}}=x_{\mathrm{IQI}}+d_{\mathrm{t}}=\nu_{1} x_{m}+\nu_{2} x_{m}^{*}+d_{\mathrm{t}}
$$

The transmitted signal undergoes a slowly varying flat Rayleigh fading channel $h \sim \mathcal{C N}(0, \lambda, 0)$. Moreover, the receiver further induces an additive distortion $d_{\mathrm{r}} \sim \mathcal{C N}\left(0, \sigma_{\mathrm{r}}^{2}, 0\right)$, resulting from the non-linear transfer function of low noise amplifier, band-pass filters, image rejection low pass filter, analog-to-digital converter and thermal noise at the receiver.

$$
y_{\mathrm{rx}}=\sqrt{p} h x_{\mathrm{tx}}+d_{\mathrm{r}}
$$

where $y_{\mathrm{rx}}$ is the received signal. Under the assumption of the normalized signal constellation with $\mathrm{E}\left\{\left|x_{m}\right|^{2}\right\}=1, p$ is related to the instantaneous signal-to-noise ratio $\mathrm{SNR}_{\text {ins }}$ as

$$
p=\frac{\sigma_{\mathrm{r}}^{2}}{|h|^{2}}\left[\frac{\mathrm{SNR}_{\mathrm{ins}}}{\left|\nu_{1}\right|^{2}+\left|\nu_{2}\right|^{2}-\mathrm{SNR}_{\mathrm{ins}} \sigma_{\mathrm{t}}^{2}}\right]
$$

However, we relax the transmit distortion constraint for the least complex open loop systems. Thus the parameter $p$, later used in Section VI, is related to the average $\mathrm{SNR} \mathrm{E}_{h}\left[\mathrm{SNR}_{i}\right]$ as

$$
p=\frac{\sigma_{\mathrm{r}}^{2}}{\lambda}\left[\frac{\mathrm{E}_{h}\left[\mathrm{SNR}_{\mathrm{ins}}\right]}{\left|\nu_{1}\right|^{2}+\left|\nu_{2}\right|^{2}}\right]
$$

The received signal $y_{\mathrm{rx}}$ suffers also from I/Q imbalance at the down-conversion stage, owing to the imperfect oscillator, phase shifter and I/Q mixer in the homodyne receiver. Similar to the transmit I/Q imbalance, receive I/Q imbalance induces WLT causing self-interference of the $y_{\mathrm{rx}}$.

$$
y=\mu_{1} y_{\mathrm{rx}}+\mu_{2} y_{\mathrm{rx}}^{*}
$$

The parameters $\mu_{1}$ and $\mu_{2}$ constitute the amplitude $a_{\mathrm{r}}$ and rotational errors $\theta_{\mathrm{r}}$ in the down-conversion process (8). For perfect receiver I/Q balance $\mu_{1}=1$ and $\mu_{2}=0$ which follows from $a_{\mathrm{r}}=1$ and $\theta_{\mathrm{r}}=0$.

$$
\mu_{1}=\left(1+a_{\mathrm{r}} e^{j \theta_{\mathrm{r}}}\right) / 2 ; \mu_{2}=1-\mu_{1}^{*}=\left(1-a_{\mathrm{r}} e^{-j \theta_{\mathrm{r}}}\right) / 2
$$

Therefore, the received signal $y$ under the presented HWIs model is given in (8). The parameter $\tilde{h}_{1}$ and $\tilde{h}_{2}$ are the modified channel gain accumulating fading characteristics as well as I/Q imbalance parameters.

$$
y=\sqrt{p} \tilde{h}_{1} x_{m}+\sqrt{p} \tilde{h}_{2} x_{m}^{*}+z ; \quad m=1,2, \ldots, M
$$

where $\tilde{h}_{1}=\mu_{1} \nu_{1} h+\mu_{2} \nu_{2}^{*} h^{*}$ and $\tilde{h}_{2}=\mu_{1} \nu_{2} h+\mu_{2} \nu_{1}^{*} h^{*}$. It is important to note that the useful signal component comprises of both the actual signal $x_{m}$ and the SI signal $x_{m}^{*}$. It is justified from the information theoretic definition of mutual information. Otherwise, treating the SI term as mere interference will result in a loss of useful information. Thus the aggregated interference term $z$ is expressed as

$$
z=\mu_{1}\left(\sqrt{p} h d_{\mathrm{t}}+d_{\mathrm{r}}\right)+\mu_{2}\left(\sqrt{p} h^{*} d_{\mathrm{t}}^{*}+d_{\mathrm{r}}^{*}\right)
$$

Theorem 1. WLT model of the transceiver I/Q imbalance transforms the proper/ symmetric transmitted signal to improper/asymmetric received signal and the proper Gaussian interference to improper Gaussian interference.

Proof: This follows from the non-zero pseudo-variance of the received signal and the accumulative interference term under HWI that are expressed, respectively, as

$$
\tilde{\sigma}_{y}^{2}=2 \tilde{h}_{1} \tilde{h}_{2}+\tilde{\sigma}_{z}^{2} \quad \tilde{\sigma}_{z}^{2}=2 \mu_{1} \mu_{2}\left(p|h|^{2} \sigma_{\mathrm{t}}^{2}+\sigma_{\mathrm{r}}^{2}\right)
$$

This work exclusively emphasizes on the transceiver I/Q imbalance pertaining to their SI distinct model. The presence of I/Q imbalance yields SI information-bearing signals and improper additive noise, which changes the system characteristics. It renders the conventional detectors as sub-optimal detectors as they are incapable of supporting the WLT structure induced by the I/Q imbalance.

\section{Optimal MAXIMUM Likelihood Receiver}

Given the equiprobable symbols and improper interference, the conventional minimum Euclidean distance (MED) detector performs sub-optimally as it fails to accommodate the nonidentical and dependent real and imaginary components of the improper noise. Therefore, we propose an optimal maximum a posteriori (MAP) detector for the presented hardware impaired system model. Consider the general signal model in (12), the useful signal component along with the fading characteristics and I/Q imbalance estimates is given by $\chi^{m}=\tilde{h}_{1} x_{m}+$ $\tilde{h}_{2} x_{m}^{*} \forall m=1,2, \ldots, M$ and $z$ is the accumulated noise component (10).

$$
y=\sqrt{p} \chi^{m}+z ; \quad m=1,2, \ldots, M
$$


For a given channel, I/Q imbalance estimates and transmitted signal $x_{m}$, the real component $y_{r}$ and imaginary component $y_{i}$ of the received signal $y$ are jointly Gaussian with PDF

$$
\begin{aligned}
& f_{y_{\mathrm{r}} y_{\mathrm{i}} \mid \chi^{m}}\left(y_{\mathrm{r}}, y_{\mathrm{i}} \mid \chi^{m}\right)=\frac{1}{2 \pi \sigma_{\mathrm{I}} \sigma_{\mathrm{Q}} \sqrt{1-\rho^{2}}} \exp \frac{-1}{2\left(1-\rho^{2}\right)} \cdots \\
& \left(\frac{\left(y_{\mathrm{r}}-\sqrt{p} \chi_{\mathrm{r}}^{m}\right)^{2}}{\sigma_{\mathrm{I}}^{2}}+\frac{\left(y_{\mathrm{i}}-\sqrt{p} \chi_{\mathrm{i}}^{m}\right)^{2}}{\sigma_{\mathrm{Q}}^{2}}-\frac{2 \rho\left(y_{\mathrm{r}}-\sqrt{p} \chi_{\mathrm{r}}^{m}\right)\left(y_{\mathrm{i}}-\sqrt{p} \chi_{\mathrm{i}}^{m}\right)}{\sigma_{\mathrm{I}} \sigma_{\mathrm{Q}}}\right) .
\end{aligned}
$$

The in-phase and quadrature components of $z$ are zero-mean Gaussian RV with variance $\sigma_{\mathrm{I}}^{2}$ and $\sigma_{\mathrm{Q}}^{2}=a_{\mathrm{r}}^{2} \sigma_{\mathrm{I}}^{2}$, where

$$
\sigma_{\mathrm{I}}^{2}=\frac{\sigma_{\mathrm{r}}^{2}}{2}+\frac{p \sigma_{\mathrm{t}}^{2}}{2}|h|^{2} \text {. }
$$

Interestingly, the noise variance not only depends on the I/Q imbalance parameter and distortion variance but also on the channel gain and SNR. Thus, increasing SNR amplifies also the corresponding noise owing to the presence of transmitter distortion. Using (10), the correlation coefficient $\rho$ between $z_{\mathrm{r}}$ and $z_{\mathrm{i}}$ simplifies to (15). The correlation only depends on the amount of rotational error induced by the receiver.

$$
\rho=\frac{\mathrm{E}\left(z_{\mathrm{r}} z_{\mathrm{i}}\right)}{\sigma_{\mathrm{I}} \sigma_{\mathrm{Q}}}=\sin \theta_{\mathrm{r}}
$$

MAP detector reduces to ML detector for the case of equiprobable symbols. Therefore, the optimal detection is based on maximizing the following conditional probability

$$
\hat{x}_{m}=\underset{m=1,2, \ldots, M}{\arg \max } f_{y_{\mathrm{r}} y_{\mathrm{i}} \mid \chi^{m}}\left(y_{\mathrm{r}}, y_{\mathrm{i}} \mid \chi^{m}\right)
$$

Using (13), the ML detector in (16) reduces to the minimization of the argument of conditional probability $f_{y_{\mathrm{r}} y_{\mathrm{i}} \mid \chi^{m}}\left(y_{\mathrm{r}}, y_{\mathrm{i}} \mid \chi^{m}\right)$.

$$
\hat{x}_{m}=\underset{m=1,2, \ldots, M}{\arg \min }\left\{\begin{array}{l}
\frac{\left(y_{\mathrm{r}}-\sqrt{p} \chi_{\mathrm{r}}^{m}\right)^{2}}{\sigma_{\mathrm{I}}^{2}}+\frac{\left(y_{\mathrm{i}}-\sqrt{p} \chi_{\mathrm{i}}^{m}\right)^{2}}{\sigma_{\mathrm{Q}}^{2}}+ \\
-\frac{2 \rho\left(y_{\mathrm{r}}-\sqrt{p} \chi_{\mathrm{r}}^{m}\right)\left(y_{\mathrm{i}}-\sqrt{p} \chi_{\mathrm{i}}^{m}\right)}{\sigma_{\mathrm{I}} \sigma_{\mathrm{Q}}}
\end{array}\right\} .
$$

Using (14) and (15), the simplified optimal ML detector is

$$
\hat{x}_{m}=\underset{m=1,2, \ldots, M}{\arg \min }\left\{\begin{array}{c}
\left(y_{\mathrm{r}}-\sqrt{p} \chi_{\mathrm{r}}^{m}\right)^{2}+\frac{1}{a_{\mathrm{R}}^{2}}\left(y_{\mathrm{i}}-\sqrt{p} \chi_{\mathrm{i}}^{m}\right)^{2}+ \\
-\frac{2 \sin \theta_{\mathrm{R}}}{a_{\mathrm{R}}}\left(y_{\mathrm{r}}-\sqrt{p} \chi_{\mathrm{r}}^{m}\right)\left(y_{\mathrm{i}}-\sqrt{p} \chi_{\mathrm{i}}^{m}\right)
\end{array}\right\} .
$$

Assuming $\tilde{h}_{\mathrm{kr}}$ and $\tilde{h}_{\mathrm{ki}}$ are the real and imaginary components of $\tilde{h}_{\mathrm{k}}$ respectively. In addition, $x_{\mathrm{r}}^{m}$ and $x_{\mathrm{i}}^{m}$ are the real and imaginary components of the transmitted symbol $x_{m}$ respectively. The useful in-phase signal component $\chi_{\mathrm{r}}^{m}$ and quadrature component $\chi_{\mathrm{i}}^{m}$ are given as

$$
\begin{aligned}
& \chi_{\mathrm{r}}^{m}=\left(\tilde{h}_{1 \mathrm{r}}+\tilde{h}_{2 \mathrm{r}}\right) x_{\mathrm{r}}^{m}+\left(\tilde{h}_{2 \mathrm{i}}-\tilde{h}_{1 \mathrm{i}}\right) x_{\mathrm{i}}^{m} \\
& \chi_{\mathrm{i}}^{m}=\left(\tilde{h}_{1 \mathrm{i}}+\tilde{h}_{2 \mathrm{i}}\right) x_{\mathrm{r}}^{m}+\left(\tilde{h}_{1 \mathrm{r}}-\tilde{h}_{2 \mathrm{r}}\right) x_{\mathrm{i}}^{m} .
\end{aligned}
$$

The optimal detector in (18) differs from the traditional MED detector for Gaussian interference pertaining to the presence of amplitude translation $a_{\mathrm{r}}$ and rotational error $\theta_{\mathrm{r}}$ caused by the receiver I/Q imbalance. The amplitude error is responsible for the non-identical real and imaginary components and the rotational error is accountable for the mutual correlation. Whereas the traditional MED detector with the assumption of circularly symmetric complex Gaussian interference is given by

$$
\hat{x}_{m}=\underset{m=1,2, \ldots, M}{\arg \min }\left|y-\sqrt{p} \chi^{m}\right|^{2},
$$

where $\chi^{m}=h x_{m}$ under ideal hardware assumption.

\section{ERROR PROBABILITY ANALYSIS}

\section{A. Pairwise Error Probability}

Symbol error probability (SEP) $P_{\mathrm{s}}^{\mathrm{e}}$ is defined as the probability of deciding $\chi^{n}$ given $\chi^{m}$ was transmitted with prior probability $\operatorname{Pr}\left(\chi^{m}\right)$ for all $m \neq n$.

$$
P_{\mathrm{s}}^{\mathrm{e}}=\sum_{m=1}^{M} \sum_{\substack{n=1 \\ n \neq m}}^{M} \operatorname{Pr}\left(\chi^{m} \rightarrow \chi^{n} \mid \chi^{m}\right) \operatorname{Pr}\left(\chi^{m}\right)
$$

where the term $\operatorname{Pr}\left(\chi^{m} \rightarrow \chi^{n} \mid \chi^{m}\right)$ is well-known as pairwise error probability (PEP). Under the assumption of equal likely symbols with equal priori $M^{-1}, P_{\mathrm{s}}^{\mathrm{e}}$ reduces to

$$
P_{\mathrm{s}}^{\mathrm{e}}=\frac{1}{M} \sum_{m=1}^{M} \sum_{\substack{n=1 \\ n \neq m}}^{M} \operatorname{Pr}\left(\chi^{m} \rightarrow \chi^{n} \mid \chi^{m}\right)
$$

At high SNR, most error events occur with neighboring symbol, where the probability of making error with neighboring symbol is higher than the error probability with a far point which is not located at minimum distance $\left(d_{\min }\right)$ from the transmitted symbol. Thus, we can upper bound symbol error probability by the PEP between two closest possibilities.

$$
P_{\mathrm{s}}^{\mathrm{e}} \leq(M-1) \operatorname{Pr}\left(\chi^{m} \rightarrow \chi^{n} \mid \chi^{m}\right),
$$

where $x_{n}$ is assumed to be the neighbor located at $d_{\min }$ from $x_{m}$. Although this is a loose upper bound as it assumes all neighbors to be located at $d_{\text {min }}$, which is valid for orthogonal modulation schemes. However, generally this is not the case in others. Therefore, we will use well-known nearest neighbor union bound (25) for the M-ary modulation.

$$
P_{\mathrm{s}}^{\mathrm{e}} \leq \psi \operatorname{Pr}\left(\chi^{m} \rightarrow \chi^{n} \mid \chi^{m}\right)
$$

where $\psi$ represents the average number of neighbors located at $d_{\text {min }}$ distance to each other. For example, for M-PSK $\psi=2$ and for M-QAM square constellation

$$
\psi=\frac{1}{M}\left[8+12(\sqrt{M}-2)+4(\sqrt{M}-2)^{2}\right] .
$$

Based on the proposed optimal ML detector, the pairwise error probability is defined by the following conditional probability

$$
\begin{aligned}
& \operatorname{Pr}\left(\chi^{m} \rightarrow \chi^{n} \mid \chi^{m}\right)= \\
& \operatorname{Pr}\left\{\begin{array}{l}
\frac{\left(y_{\mathrm{r}}-\sqrt{p} \chi_{\mathrm{r}}^{m}\right)^{2}}{\sigma_{\mathrm{I}}^{2}}+\frac{\left(y_{\mathrm{i}}-\sqrt{p} \chi_{\mathrm{i}}^{m}\right)^{2}}{\sigma_{\mathrm{Q}}^{2}}-\frac{2 \rho\left(y_{\mathrm{r}}-\sqrt{p} \chi_{\mathrm{r}}^{m}\right)\left(y_{\mathrm{i}}-\sqrt{p} \chi_{\mathrm{i}}^{m}\right)}{\sigma_{\mathrm{I}} \sigma_{\mathrm{Q}}}> \\
\frac{\left(y_{\mathrm{r}}-\sqrt{p} \chi_{\mathrm{r}}^{n}\right)^{2}}{\sigma_{\mathrm{I}}^{2}}+\frac{\left(y_{\mathrm{i}}-\sqrt{p} \chi_{\mathrm{i}}^{n}\right)^{2}}{\sigma_{\mathrm{Q}}^{2}}-\frac{2 \rho\left(y_{\mathrm{r}}-\sqrt{p} \chi_{\mathrm{r}}^{n}\right)\left(y_{\mathrm{i}}-\sqrt{p} \chi_{\mathrm{i}}^{n}\right)}{\sigma_{\mathrm{I}} \sigma_{\mathrm{Q}}}
\end{array}\right\}
\end{aligned}
$$

Using the fact that $x_{m}$ was transmitted, and replacing all occurrences of $y_{\mathrm{r}}$ with $\sqrt{p} \chi_{\mathrm{r}}^{m}+z_{\mathrm{r}}$ and $y_{\mathrm{i}}$ with $\sqrt{p} \chi_{\mathrm{i}}^{m}+z_{\mathrm{i}}$, we obtain (28) after some simplification

$$
\mathrm{PEP}=\operatorname{Pr}\left\{\eta>\frac{p \zeta_{\mathrm{r}}^{2}}{\sigma_{\mathrm{I}}^{2}}+\frac{p \zeta_{\mathrm{i}}^{2}}{\sigma_{\mathrm{Q}}^{2}}-\frac{2 p \rho \zeta_{\mathrm{r}} \zeta_{\mathrm{i}}}{\sigma_{\mathrm{I}} \sigma_{\mathrm{Q}}}\right\}
$$

where $\zeta_{\mathrm{r}}=\chi_{\mathrm{r}}{ }^{m}-\chi_{\mathrm{r}}{ }^{n}$ and $\zeta_{\mathrm{i}}=\chi_{\mathrm{i}}{ }^{m}-\chi_{\mathrm{i}}{ }^{n}$ are the distances between real and imaginary components of the transmitted and received useful signal component in an error event respectively. Furthermore, $\eta$ is obtained by the superposition of $z_{\mathrm{r}}$ and $z_{\mathrm{i}}$.

$$
\eta=\left(\frac{2 \rho \zeta_{\mathrm{i}}}{\sigma_{\mathrm{I}} \sigma_{\mathrm{Q}}}-\frac{2 \zeta_{\mathrm{r}}}{\sigma_{\mathrm{I}}^{2}}\right) \sqrt{p} z_{\mathrm{r}}+\left(\frac{2 \rho \zeta_{\mathrm{r}}}{\sigma_{\mathrm{I}} \sigma_{\mathrm{Q}}}-\frac{2 \zeta_{\mathrm{i}}}{\sigma_{\mathrm{Q}}^{2}}\right) \sqrt{p} z_{\mathrm{i}}
$$


The zero-mean Gaussian random variable $\eta$ is distributed with variance $\sigma_{\eta}^{2}$, given as

$$
\sigma_{\eta}^{2}=4\left(1-\rho^{2}\right)\left[\frac{p \zeta_{\mathrm{r}}^{2}}{\sigma_{\mathrm{I}}^{2}}+\frac{p \zeta_{\mathrm{i}}^{2}}{\sigma_{\mathrm{Q}}^{2}}-\frac{2 p \rho \zeta_{\mathrm{r}} \zeta_{\mathrm{i}}}{\sigma_{\mathrm{I}} \sigma_{\mathrm{Q}}}\right]
$$

Thus the probability in (28), is the complementary CDF of the Gaussian random variable $\eta$ and is given by

$$
\mathrm{PEP}=Q \sqrt{\frac{1}{4\left(1-\rho^{2}\right)}\left(\frac{p \zeta_{\mathrm{r}}^{2}}{\sigma_{\mathrm{I}}^{2}}+\frac{p \zeta_{\mathrm{i}}^{2}}{\sigma_{\mathrm{Q}}^{2}}-\frac{2 p \rho \zeta_{\mathrm{r}} \zeta_{\mathrm{i}}}{\sigma_{\mathrm{I}} \sigma_{\mathrm{Q}}}\right)}
$$

The instantaneous PEP expression depends on the distance between the transmitted and received erroneous signal component under Rayleigh fading, I/Q imbalance and transceiver additive distortions. It further relies on the average SNR and statistical characteristics of the non-identical and dependent I/Q phase improper interference components.

\section{B. Asymptotic Analysis}

The dependence of the statistical characteristics of the improper interference components on the average SNR motivates us to analyze the asymptotic behavior of the PEP expression with respect to the parameter $p$. Substituting (14) and (15) in (31) yields

$$
\mathrm{PEP}=Q\left(\sqrt{\frac{a_{\mathrm{r}}^{2} p \zeta_{\mathrm{r}}^{2}+p \zeta_{\mathrm{i}}^{2}-2 a_{\mathrm{r}} p \sin \theta_{\mathrm{r}} \zeta_{\mathrm{r}} \zeta_{\mathrm{i}}}{2 a_{\mathrm{r}}^{2} \cos ^{2} \theta_{\mathrm{r}}\left(\sigma_{\mathrm{r}}^{2}+p \sigma_{\mathrm{t}}^{2}|h|^{2}\right)}}\right)
$$

Based on the average SNR relation in (6), the asymptotic behavior of the instantaneous PEP depicts an irreducible error floor. It is important to note that out of all the above mentioned impairments, the transmitter distortions are mainly responsible for the error floor.

$$
\lim _{p \rightarrow \infty} \mathrm{PEP}=Q\left(\sqrt{\frac{a_{\mathrm{r}}^{2} \zeta_{\mathrm{r}}^{2}+\zeta_{\mathrm{i}}^{2}-2 a_{\mathrm{r}} \sin \theta_{\mathrm{r}} \zeta_{\mathrm{r}} \zeta_{\mathrm{i}}}{2 a_{\mathrm{r}}^{2} \sigma_{\mathrm{t}}^{2} \cos ^{2} \theta_{\mathrm{r}}|h|^{2}}}\right)
$$

Intuitively, increasing SNR also increases the transmitter distortions. Thus rendering it ineffective to reduce error probabilities of the non-zero transmitter distortion systems. From (32), it is evident that assuming $\sigma_{\mathrm{t}}^{2}=0$ will result in $\lim _{p \rightarrow \infty} \mathrm{PEP}=0$.

\section{Average Probability of ERror}

\section{A. HWI Transmitter and Receiver}

Consider the generalized system model under transmitter and receiver I/Q imbalance as well as the transmitter and receiver additive distortions. Pairwise probability of such adopted system is given by (32). Average pairwise error probability $\left(A_{\mathrm{p}}\right)$ of the generalized model is investigated with the assumption that $\theta_{\mathrm{r}} \leq 5^{0}$, which is a valid assumption for the I/Q imbalance rotational errors as experimentally investigated in [6]. This assumption applies that $\sin \theta_{\mathrm{r}} \approx 0$, therefore the PEP expression reduces to

$$
\mathrm{PEP}=Q\left(\sqrt{\frac{\alpha h_{\mathrm{r}}^{2}+\beta h_{\mathrm{i}}^{2}}{1+\gamma\left(h_{\mathrm{r}}^{2}+h_{\mathrm{i}}^{2}\right)}}\right)
$$

where $\alpha$ and $\beta$ are defined as

$$
\alpha=\frac{p\left(a_{\mathrm{r}}^{2} \varsigma_{1}^{2}+\varsigma_{2}^{2}\right)}{2 \sigma_{\mathrm{r}}^{2} a_{\mathrm{r}}^{2} \cos ^{2} \theta_{\mathrm{r}}}, \quad \beta=\frac{p\left(a_{\mathrm{r}}^{2} \varsigma_{3}^{2}+\varsigma_{4}^{2}\right)}{2 \sigma_{\mathrm{r}}^{2} a_{\mathrm{r}}^{2} \cos ^{2} \theta_{\mathrm{r}}}
$$

In addition, $\gamma$ is the scaled ratio of transmitter distortion variance to receiver distortion variance i.e. $\gamma=p \sigma_{\mathrm{t}}^{2} / \sigma_{\mathrm{r}}^{2}$. Moreover, the $\varsigma_{s} \forall s \in\{1,2,3,4\}$ are obtained using I/Q imbalance estimations and the respective modulation scheme characteristics as $\varsigma_{1}=\xi_{\mathrm{r}}, \varsigma_{2}=a_{\mathrm{t}} a_{\mathrm{r}} \cos \theta_{\mathrm{t}} \cos \theta_{\mathrm{r}} \xi_{\mathrm{i}}, \varsigma_{3}=$ $a_{\mathrm{t}} \cos \theta_{\mathrm{t}} \xi_{\mathrm{i}}, \quad \varsigma_{4}=a_{\mathrm{r}} \cos \theta_{\mathrm{r}} \xi_{\mathrm{r}}$. They particularly depend on the symbol separation between two closest possibilities with $\xi_{\mathrm{r}}=\left(x_{\mathrm{r}}^{m}-x_{\mathrm{r}}^{n}\right)$ being the separation between the real components and $\xi_{\mathrm{i}}=\left(x_{\mathrm{i}}^{m}-x_{\mathrm{i}}^{n}\right)$ being the separation between the corresponding imaginary components. Let us denote the argument of the Q-function in (34) as

$$
\vartheta=\frac{\alpha h_{\mathrm{r}}^{2}+\beta h_{\mathrm{i}}^{2}}{1+\gamma\left(h_{\mathrm{r}}^{2}+h_{\mathrm{i}}^{2}\right)}
$$

Under the assumption of the Rayleigh fading channel, the random variable $\vartheta$ for a given signal constellation and I/Q imbalance parameters estimates has a cumulative density function (CDF) $F_{\vartheta}(\vartheta)$ that is derived in Appendix A as

$$
F_{\vartheta}(\vartheta)=\frac{1}{k_{1}(\vartheta) k_{3}(\vartheta)} \int_{0}^{\vartheta k_{3}(\vartheta)} e^{-t \frac{k_{2}(\vartheta)}{k_{3}(\vartheta)}} \mathcal{I}_{0}\{t\} d t ; 0 \leq \vartheta \leq \delta
$$

where $\delta=\max (\alpha, \beta) / \gamma$ and $\mathcal{I}_{0}\{t\}$ is the zero-order modified Bessel function. Furthermore $k_{1}(\vartheta), k_{2}(\vartheta)$ and $k_{3}(\vartheta)$ are expressed, respectively, as

$$
\begin{gathered}
k_{1}(\vartheta)=\lambda \sqrt{(\alpha-\gamma \vartheta)(\beta-\gamma \vartheta)} \\
k_{2}(\vartheta)=\frac{\lambda(\alpha+\beta-2 \gamma \vartheta)}{2\left[k_{1}(\vartheta)\right]^{2}}, \quad k_{3}(\vartheta)=\frac{\lambda(\beta-\alpha)}{2\left[k_{1}(\vartheta)\right]^{2}}
\end{gathered}
$$

Distribution function $F_{\vartheta}(\vartheta)$ in (37) can be obtained in a closed form using the following power series representation

$$
\mathcal{I}_{0}\{t\}=\sum_{m=0}^{\infty} \frac{t^{2 m}}{[m !]^{2} 2^{2 m}}
$$

Based on the uniform convergence, swapping the order of integral and summation yields the closed form distribution function $F_{\vartheta}(\vartheta)$ as

$$
F_{\vartheta}(\vartheta)=\frac{1}{k_{1}(\vartheta)} \sum_{m=0}^{\infty} \frac{2^{-2 m} k_{3}(\vartheta)^{2 m}}{[m !]^{2} k_{2}(\vartheta)^{2 m+1}}\left[2 m !-\Gamma\left(2 m+1, \vartheta k_{2}(\vartheta)\right)\right]
$$

where $\Gamma(a, x)$ is the upper incomplete gamma function. $A_{\mathrm{p}}$ is then obtained by averaging the PEP expression in (34) with respect to $\vartheta$ i.e, $A_{\mathrm{p}}=\mathrm{E}_{\vartheta}[Q(\sqrt{\vartheta})]$. The application of Craig's formula to represent Q-function is not very insightful in the presented scenario as it yields intractable analysis. Therefore, relying on the Chernoff bound of the Q-function $\left(Q(x) \leq 0.5 \exp \left(-x^{2} / 2\right) \forall x \geq 0\right)$ yields

$$
A_{\mathrm{p}} \leq \frac{1}{2} \int_{0}^{\delta} e^{-\frac{\vartheta}{2}} f_{\vartheta}(\vartheta) d \vartheta=\frac{1}{2} e^{-\frac{\delta}{2}}+\frac{1}{4} \int_{0}^{\delta} e^{-\frac{\vartheta}{2}} F_{\vartheta}(\vartheta) d \vartheta
$$

The equality in (42) is obtained after applying integration by parts, which enables us to represent the presented bound in terms of $\operatorname{CDF} F_{\vartheta}(\vartheta)$. Average PEP bound can be accurately 
evaluated using numerical integration techniques such as Gaussian quadrature. Existence of the definite integral with finite limits motivates us to employ the popular $n^{\text {th }}$ order Legendre orthogonal polynomial $P_{n}(t)$ [15, (2.5.49-51)]. Assuming $s(\vartheta)=e^{-\frac{\vartheta}{2}} F_{\vartheta}(\vartheta)$, we obtain the required form by using the given parametrization,

$$
A_{\mathrm{p}} \leq \frac{1}{2} e^{-\frac{\delta}{2}}+\frac{\delta}{8} \int_{-1}^{+1} s(0.5 \delta(t+1)) d t .
$$

Gaussian quadrature enables us to numerically approximate the integral given in (43) with weights $\omega_{k}$ and s-function evaluation at the corresponding instances.

$$
A_{\mathrm{p}} \leq \frac{1}{2} e^{-\frac{\delta}{2}}+\frac{\delta}{8}\left[\sum_{k=1}^{n} \omega_{k} s\left(0.5 \delta\left(t_{k}+1\right)\right)+R_{n}\right]
$$

where the node $t_{k}$ is the $k^{\text {th }}$ zero of the $n^{\text {th }}$ - order Legendre polynomial $\mathrm{P}_{n}(t)$ and the corresponding weights are obtained using [15, (25.4.30)].

$$
\omega_{k}=\frac{2}{\left(1-t_{k}^{2}\right)\left[\mathrm{P}_{n}{ }^{(1)}\left(t_{k}\right)\right]^{2}}
$$

The approximation error is given by residual term $R_{n}$ [15, (25.4.30)], which decreases significantly with the increasing order of the Legendre polynomial.

$$
R_{n}=\frac{\delta^{2 n+1}(n !)^{4}}{(2 n+1)[(2 n) !]^{3}} f^{(2 n)}(\phi) \quad ;-1<\phi<1
$$

This numerical integration technique enables us to precisely evaluate the intractable integral in (42) as given in (44).

1) Zero-Distortion Transmitter: In this subsection, we propose a closed form expression for the average PEP expression of the adopted system model under transmitter and receiver I/Q imbalance along with the thermal noise at receiver but negligible transmitter distortion. This simplifies the PEP expression in (34) to $\mathrm{PEP}=Q(\sqrt{\varrho})$ where $\varrho$ is defined as

$$
\varrho=\alpha h_{\mathrm{r}}^{2}+\beta h_{\mathrm{i}}^{2}
$$

Following similar steps as in Appendix B, the PDF of $f_{\varrho}(\varrho)$ for the Rayleigh fading channel is given as

$f_{\varrho}(\varrho)=\frac{1}{\lambda \sqrt{\alpha \beta}} e^{-\frac{\varrho}{2 \lambda}\left(\frac{1}{\alpha}+\frac{1}{\beta}\right)} \mathrm{I}_{0}\left\{\frac{\varrho}{2 \lambda}\left(\frac{1}{\alpha}-\frac{1}{\beta}\right)\right\} ; \varrho \geq 0$

Employing the Chernoff bound on the Q-function yields the following bound on the average PEP $A_{p}=\mathrm{E}_{\varrho}[Q(\sqrt{\varrho})]$.

$$
A_{\mathrm{p}} \leq \frac{1}{2} \int_{0}^{\infty} e^{-\frac{\varrho}{2}} f_{\varrho}(\varrho) d \varrho
$$

Based on the uniform convergence, the interchangeable integral and summation enable us to evaluate the closed form expression for the $A_{\mathrm{p}}$ bound as shown in Appendix B.

$$
A_{\mathrm{p}} \leq \frac{\sqrt{\alpha \beta}}{|\beta-\alpha|} \sum_{m=0}^{\infty} \frac{(2 m) !}{[m !]^{2} 2^{2 m}}\left[\frac{|\beta-\alpha|}{(\alpha+\beta+\alpha \beta \lambda)}\right]^{2 m+1}
$$

\section{B. HWI Receiver}

This section deals with the receiver I/Q imbalance under the assumption of minimal transmitter I/Q imbalance. An example of such scenario is the cellular downlink case when the BTS employs sophisticated signal processing techniques rendering minimal I/Q imbalance. However, the mobile station employs homodyne RF front-end architecture to assist compactness and energy efficiency. In such scenario, the system only suffers from the receiver I/Q imbalance.

In the presence of receiver thermal noise, we further consider two scenarios with and without transmitter distortion to evaluate average PEP. In the first case, system suffers from receiver I/Q imbalance as well as non-trivial transmitter and receiver distortions. In this case the $A_{\mathrm{p}}$ has the same form as given in eqs. (42) to (44) with $F_{\vartheta}(\vartheta)$ and the corresponding parameters are given in Equations (37) to (41). However, the parameters $\alpha$ and $\beta$ are now defined, respectively, as

$$
\alpha=\frac{p\left(\xi_{\mathrm{r}}^{2}+\cos ^{2} \theta_{\mathrm{r}} \xi_{\mathrm{i}}^{2}\right)}{2 \sigma_{\mathrm{r}}^{2} \cos ^{2} \theta_{\mathrm{r}}}, \quad \beta=\frac{p\left(\xi_{\mathrm{i}}^{2}+\cos ^{2} \theta_{\mathrm{r}} \xi_{\mathrm{r}}^{2}\right)}{2 \sigma_{\mathrm{r}}^{2} \cos ^{2} \theta_{\mathrm{r}}}
$$

In the second scenario, when the transmitter exhibits negligible transmit distortion, the receiver I/Q imbalanced system follows the same closed form bound as given in (48) with the corresponding parameters $\alpha$ and $\beta$ as presented in (51).

\section{HWI Transmitter}

Given the scenario of negligible I/Q imbalance at the receiver, the PEP expression in (34) reduces to

$$
\mathrm{PEP}=Q\left(\sqrt{\frac{\alpha|h|^{2}}{1+\gamma|h|^{2}}}\right)=Q(\sqrt{\Omega})
$$

where $\alpha=\frac{p}{2 \sigma_{\mathrm{r}}^{2}}\left(\tau_{1}^{2}+\tau_{2}^{2}\right), \tau_{1}=\xi_{\mathrm{r}}$ and $\tau_{2}=\left(a_{\mathrm{t}} \sin \theta_{\mathrm{t}}\right) \xi_{\mathrm{r}}+$ $\left(a_{\mathrm{t}} \cos \theta_{\mathrm{t}}\right) \xi_{\mathrm{i}}$. Recall that $\xi_{\mathrm{r}}$ and $\xi_{\mathrm{i}}$ are the separation between real and imaginary components of the two closest possibilities in a given signal constellation respectively. To evaluate the average PEP, we first need to investigate the PDF of $\Omega$ that is derived in Appendix $\mathrm{C}$ as

$$
f_{\Omega}(\Omega)=\frac{\alpha \lambda}{(\alpha-\gamma \Omega)^{2}} e^{\frac{-\lambda \Omega}{\alpha-\gamma \Omega}} ; \quad 0 \leq \Omega \leq \alpha / \gamma
$$

Therefore, using the Craig representation of the Q-function, the average PEP of the transmitter only I/Q imbalance reduces to

$$
A_{\mathrm{p}}=\frac{1}{\pi} \int_{0}^{\alpha / \gamma} \int_{0}^{\pi / 2} e^{-\frac{\Omega}{2 \sin ^{2} \phi}} \frac{\alpha \lambda}{(\alpha-\gamma \Omega)^{2}} e^{\frac{-\lambda \Omega}{\alpha-\gamma \Omega}} d \phi d \Omega
$$

The average PEP is upper bounded using the Chernoff bound as

$$
A_{\mathrm{p}} \leq \frac{\alpha \lambda}{2} \int_{0}^{\alpha / \gamma} \frac{e^{\frac{\gamma \Omega^{2}-(\alpha+2 \lambda) \Omega}{2(\alpha-\gamma \Omega)}}}{(\alpha-\gamma \Omega)^{2}} d \Omega
$$

Again, we employ Gaussian quadrature numerical integration to evaluate the presented upper bound using Legendre polynomial, which simplifies the average PEP as

$$
A_{\mathrm{p}} \leq \frac{\lambda}{\gamma} \int_{-1}^{+1} g(t) d t=\frac{\lambda}{\gamma} \sum_{k=1}^{n} \omega_{k} g\left(t_{k}\right)+R_{n}
$$




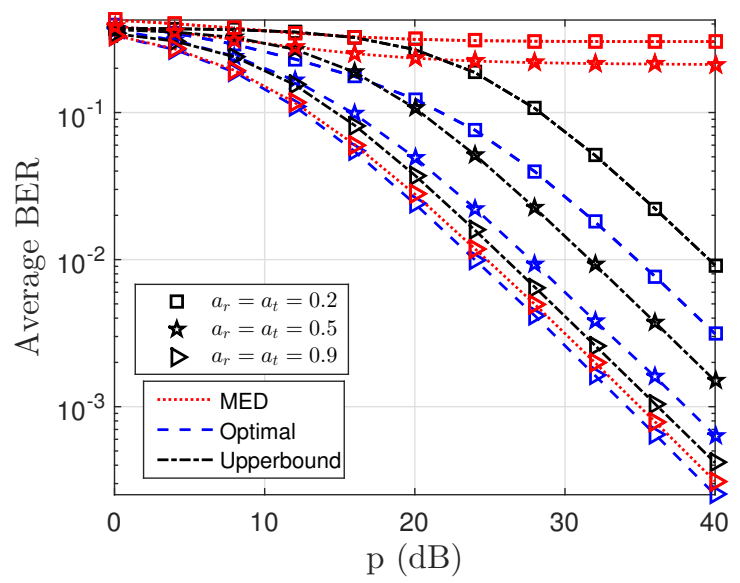

Fig. 1. System with transmitter and receiver I/Q imbalance

where the nodes $t_{k}$ are the zeros of $n^{\text {th }}-$ order Legendre polynomial and the weights $\omega_{k}$ and the residual term $R_{n}$ are defined in (45) and (46) respectively. In addition, the function $g(t)$ is derived from the integrand in (55) by choosing the parametrization $t=-1+2 \Omega \gamma / \alpha$ and is given as

$$
g(t)=\frac{1}{(t-1)^{2}} \exp \left(\frac{-\alpha(t+1)}{4 \gamma}+\frac{\lambda(t+1)}{\gamma(t-1)}\right)
$$

From (25), the average symbol error probability is thus upper bounded by the average pairwise error probability as $P_{\mathrm{s}-\text { avg }}^{\mathrm{e}} \leq$ $\psi A_{\mathrm{p}}$. Similarly, the average bit error rate can be derived as $P_{\mathrm{b}-\text { avg }}^{\mathrm{e}} \leq \psi A_{\mathrm{p}} / \log _{2} \mathrm{M}$ assuming gray coding. The average PEP $A_{\mathrm{p}}$ expressions are given in (43) and (50) for the system under dual I/Q imbalance with and without transmit distortion respectively. The same results hold for the system with only receiver I/Q imbalance with and without transmit distortion given modified parameters $\alpha$ and $\beta$ is (51). Similarly, the average PEP expression for the system with only transmitter $\mathrm{I} / \mathrm{Q}$ imbalance is proposed in (56).

\section{NumericAl RESUlts}

In this section, we numerically investigate the performance degradation of the proposed HWI system model where I/Q imbalance results in WLT and self-interference. Furthermore, we analyze the performance of the optimal detector relative to the performance of conventional MED detector in the presence of improper additive noise. The derived analytical expressions of the average probability of bit error are validated only for grey coded 16-QAM modulation for brevity. Firstly, we analyze the system under dual I/Q imbalance along with the thermal noise at the receiver. Figure 1 represents the performance gain of the optimal detector relative to the MED detector as well as the derived Chernoff bound on the average probability of bit error. We investigate average BER for the average SNR ranging from $0-40 \mathrm{~dB}$ for three different symmetric amplitude I/Q imbalance estimates $a_{\mathrm{t}}=a_{\mathrm{r}}=0.2,0.5 \& 0.9$. In addition we assume $\theta_{\mathrm{t}}=\theta_{\mathrm{r}}=4^{0}, \sigma_{\mathrm{r}}^{2}=1, \lambda=1$ and $\psi=3$ for the normalized 16-QAM modulation.

Evidently, I/Q imbalance amplitude variations degrade system performance exhibiting increased error probability. We can conclude the optimal detection of the proposed detector as compared to the MED detector with all amplitude

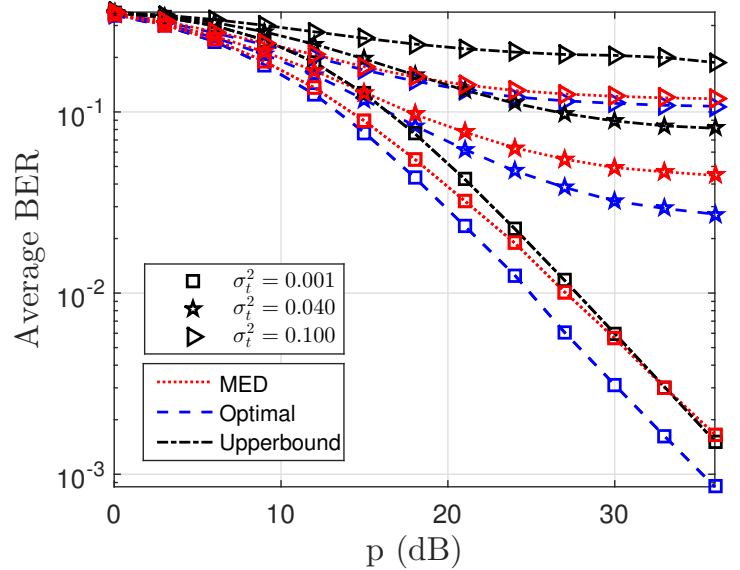

Fig. 2. System with transmitter I/Q imbalance and distortions

variations as shown in Figure 1. Moreover, MED detector exhibits error floor whereas the optimal detector effectively reduces error probability with increasing SNR. Furthermore, the derived closed form Chernoff bound is in agreement with the average BER trend and becomes tighter with decreasing impairment level.

Secondly, the average BER of the system with HWI transmitter (I/Q imbalance and additive distortion) in the absence of receiver I/Q imbalance is investigated with varying SNR for various transmit distortion variances in Figure 2. We assume transmit I/Q imbalance estimates $a_{\mathrm{t}}=0.8, \theta_{\mathrm{t}}=4$ and receiver thermal noise $\sigma_{\mathrm{r}}^{2}=1$ along with unit variance transmit signal and channel coefficient $h$ with $\psi=3$ for the normalized 16QAM modulation. The amplitude and rotational error estimates are assumed according to the experimental investigations in [6]. Clearly, the optimal detector outperforms MED detector and increasing $\sigma_{\mathrm{t}}^{2}$ severely degrades overall error performance. Interestingly, the presence of the transmit distortion creates error floor not only for the sub-optimal MED detector but also for the optimal detector. This behavior is in perfect harmony with the proposed asymptotic analysis in the presence of transmit distortion. Therefore, increasing SNR amplifies transmit distortion as well and thus rendering it ineffective to reduce error probability. The Chernoff bound is examined using the proposed Gaussian quadrature numerical integration employing 25 - order Legendre polynomial. Analysis depict that employing $10-$ order $P_{n}(t)$ exhibits promising results for SNR ranging from $0 \mathrm{~dB}$ to $25 \mathrm{~dB}$ and $15-$ order $P_{n}(t)$ exhibits promising results for SNR ranging from $0 \mathrm{~dB}$ to $30 \mathrm{~dB}$. Therefore, this depicts a trade-off between accurate bound and computational complexity at higher SNR.

\section{CONCLUSION}

In this paper, we demonstrate the significance of incorporating HWIs in accurate system modeling and analysis. We mainly focus on the detailed modeling of I/Q imbalance pertaining to its WLT model. We propose an optimal detector which incorporates improper interference characteristics. Then, we analyze its performance gain in terms of average error probability compared with the traditional MED detector. Interestingly, I/Q imbalance amplitude errors dominate rotational errors however they mutually result in SI signals. Surprisingly, 
both transmitter and receiver I/Q imbalance render SI information bearing signal whereas only receiver I/Q imbalance is responsible for transforming AWGN to improper noise. On the other hand, transmitter distortion is subject to channel fading while the receiver added impairment is not. Analytical results have been validated using simulations. The derived Chernoff bounds are in agreement with the simulated average BER trend and are significantly tight for lower impairments levels.

\section{APPENDIX A \\ DERIVATION OF $F_{\vartheta}(\vartheta)$}

Consider the Rayleigh fading channel where real and imaginary components of the channel coefficient are distributed as $h_{j} \sim \mathcal{C N}(0, \lambda / 2,0) ; j \in\{r, i\}$. This implies $h_{j}^{2}$ to be distributed as unnormalized chi-square variable with first degree of freedom with $f_{h_{j}^{2}}(x) \sim \frac{1}{\sqrt{\pi \lambda x}} e^{\frac{-x}{\lambda}} ; x \geq 0 ;$. Using (36), the CDF of $\vartheta$ is defined as $F_{\vartheta}(\vartheta)=\operatorname{Pr}\left\{\frac{\alpha h_{\mathrm{r}}^{2}+\beta h_{\mathrm{i}}^{2}}{1+\gamma\left(h_{\mathrm{r}}^{2}+h_{\mathrm{i}}^{2}\right)} \leq \vartheta\right\}$ which can also be represented as the probability of the event $F_{\vartheta}(\vartheta)=\operatorname{Pr}\left\{(\alpha-\gamma \vartheta) h_{\mathrm{r}}^{2}+(\beta-\gamma \vartheta) h_{\mathrm{i}}^{2} \leq \vartheta\right\}=\operatorname{Pr}\{S \leq \vartheta\}$

The PDF of $(\alpha-\gamma \vartheta) h_{\mathrm{r}}^{2}$ and $(\beta-\gamma \vartheta) h_{\mathrm{i}}^{2}$ is evaluated after careful transformation. Next, we convolve the derived PDFs under the assumption of uncorrelated real and imaginary channel coefficients. Then employing Mathematica integrator to compute the convolution, we obtain

$$
f_{S}(s)=\frac{1}{k_{1}(\vartheta)} e^{-s k_{2}(\vartheta)} \mathcal{I}_{0}\left\{s k_{3}(\vartheta)\right\} ; s \geq 0
$$

From (58), the distribution function $F_{\vartheta}(\vartheta)$ is given as

$$
F_{\vartheta}(\vartheta)=\frac{1}{k_{1}(\vartheta)} \int_{0}^{\vartheta} e^{-s k_{2}(\vartheta)} \mathcal{I}_{0}\left\{s k_{3}(\vartheta)\right\} d s
$$

Eventually, substituting $t=s k_{3}(\vartheta)$ yields the desired $F_{\vartheta}(\vartheta)$ expression as given in (37).

\section{APPENDIX B Chernoff Bound on $A_{\mathrm{p}}$}

Substituting $f_{\varrho}(\varrho)$ from (48) to (49) yields

$A_{\mathrm{p}} \leq \frac{1}{2 \lambda \sqrt{\alpha \beta}} \int_{0}^{\infty} e^{-\frac{\varrho}{2 \lambda}\left(\lambda+\frac{1}{\alpha}+\frac{1}{\beta}\right)} \mathrm{I}_{0}\left\{\frac{\varrho}{2 \lambda}\left(\frac{1}{\alpha}-\frac{1}{\beta}\right)\right\} d \varrho$

Substituting $t=\varrho(\beta-\alpha) /(2 \lambda \alpha \beta)$ yields

$$
A_{\mathrm{p}} \leq \frac{\sqrt{\alpha \beta}}{|\beta-\alpha|} \int_{0}^{\infty} e^{-\frac{t(\alpha+\beta+\alpha \beta \lambda)}{|\beta-\alpha|}} \mathcal{I}_{0}\{t\} d t
$$

Using the power series representation of $\mathrm{I}_{0}\{\mathrm{t}\}$ from (40) in (62) and swapping integration and summation based on the uniform convergence yields

$$
A_{\mathrm{p}} \leq \frac{\sqrt{\alpha \beta}}{|\beta-\alpha|} \sum_{m=0}^{\infty} \frac{1}{[m !]^{2} 2^{2 m}} \int_{0}^{\infty} e^{-\frac{t(\alpha+\beta+\alpha \beta \lambda)}{|\beta-\alpha|}} t^{2 m} d t
$$

Finally utilizing the well-known integral substitution (64) produces the desired closed form expression as given in (50).

$$
\int_{0}^{\infty} x^{n} e^{-a x} d x=\frac{n !}{a^{n+1}}
$$

\section{APPENDIX C \\ DERIVATION OF $f_{\Omega}(\Omega)$}

Using (52), the CDF of $\Omega$ is

$F_{\Omega}(\Omega)=\operatorname{Pr}\left\{\frac{\alpha|h|^{2}}{1+\gamma|h|^{2}} \leq \Omega\right\}=\operatorname{Pr}\left\{(\alpha-\gamma \Omega)|h|^{2} \leq \Omega\right\}$

Rayleigh fading channel assumption yields $f_{|h|^{2}}(x)=\lambda e^{-\lambda x}$. This implies $(\alpha-\gamma \Omega)|h|^{2} \sim \operatorname{Exp}[(\alpha-\gamma \Omega) / \lambda]$ Therefore, the probability in (65) turns out to be $F_{\Omega}(\Omega)=1-e^{\frac{-\lambda \Omega}{\alpha-\gamma \Omega}}$, that can be differentiated giving $f_{\Omega}(\Omega)$ as given in (53).

\section{REFERENCES}

[1] B. Razavi, "Design considerations for direct-conversion receivers," IEEE Trans. Circuits Syst. II, vol. 44, no. 6, pp. 428-435, Jun. 1997.

[2] A. A. Abidi, "Direct-conversion radio transceivers for digital communications," IEEE J. Solid-State Circuits, vol. 30, no. 12, pp. 1399-1410, Dec. 1995.

[3] S. Buzzi, I. Chih-Lin, T. E. Klein, H. V. Poor, C. Yang, and A. Zappone, "A survey of energy-efficient techniques for $5 \mathrm{G}$ networks and challenges ahead," IEEE J. Sel. Areas Commun., vol. 34, no. 4, pp. 697-709, Apr. 2016.

[4] E. Hossain and M. Hasan, "5G cellular: key enabling technologies and research challenges," IEEE Trans. Instrum. Meas., vol. 18, no. 3, pp. 11-21, May. 2015.

[5] H. Mehrpouyan, M. Matthaiou, R. Wang, G. K. Karagiannidis, and Y. Hua, "Hybrid millimeter-wave systems: A novel paradigm for HetNets," IEEE Commun. Mag., vol. 53, no. 1, pp. 216-221, Jan. 2015.

[6] T. Schenk, $R F$ imperfections in high-rate wireless systems: impact and digital compensation. Springer Science \& Business Media, 2008.

[7] R. Krishnan, On the Impact of Phase Noise in Communication Systems-Performance Analysis and Algorithms. Chalmers University of Technology, Apr. 2015.

[8] R. Ghods, C. Jeon, A. Maleki, and C. Studer, "Optimal large-mimo data detection with transmit impairments," in 53rd Annual Allerton Conf. Commun. Control Comput. (Allerton). IEEE, Oct. 2015, pp. 12111218.

[9] S. Javed, O. Amin, S. S. Ikki, and M. S. Alouini, "Impact of improper Gaussian signaling on hardware impaired systems," in Proc. 2017 IEEE Intern. Conf. Commun. (ICC 2017), May 2017, pp. 1-6.

[10] S. Javed, O. Amin, S. S. Ikki, and M.-S. Alouini, "Asymmetric hardware distortions in receive diversity systems: Outage performance analysis," IEEE Access, vol. 5, pp. 4492-4504, Feb. 2017.

[11] H. D. Nguyen, R. Zhang, and S. Sun, "Improper signaling for symbol error rate minimization in $k$-user interference channel," IEEE Trans. Commun., vol. 63, no. 3, pp. 857-869, Jan 2015.

[12] F. D. Neeser and J. L. Massey, "Proper complex random processes with applications to information theory," IEEE Trans. Inf. Theory, vol. 39, no. 4, pp. 1293-1302, Jul. 1993.

[13] A. Behzad, K. A. Carter, H.-M. Chien, S. Wu, M.-A. Pan, C. P. Lee, Q. Li, J. C. Leete, S. Au, M. S. Kappes et al., "A fully integrated MIMO multiband direct conversion CMOS transceiver for wlan applications (802.11 n)," IEEE J. Solid-State Circuits, vol. 42, no. 12, pp. 27952808, Nov. 2007.

[14] E. Björnson, M. Matthaiou, and M. Debbah, "A new look at dual-hop relaying: Performance limits with hardware impairments," IEEE Trans. Commun., vol. 61, no. 11, pp. 4512-4525, Nov. 2013.

[15] M. Abramowitz and I. A. Stegun, Handbook of mathematical functions: with formulas, graphs, and mathematical tables. Courier Corporation, 1964, vol. 55. 